\title{
Application of Normative Occipital Condyle-C1 Interval Measurements to Detect Atlanto-Occipital Injury in Children
}

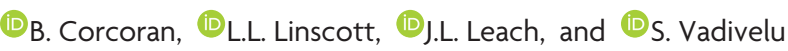

\begin{abstract}
BACKGROUND AND PURPOSE: Prior studies have found that widening or asymmetry of the occipital condyle-C1 interval on CT is a sensitive and specific marker for atlanto-occipital dislocation. Previously reported abnormal occipital condyle- $\mathrm{Cl}$ interval values are not age-specific, possibly leading to false-positive findings in younger children, in whom this joint space is normally larger than that in adults. This study assesses the utility of applying age-specific normative occipital condyle- $\mathrm{Cl}$ interval ranges to documented cases of atlantooccipital injury compared with previously reported abnormal cutoff values.
\end{abstract}

MATERIALS AND METHODS: Retrospective review of CT and MR imaging of 14 subjects with atlanto-occipital injury was performed, and occipital condyle- $\mathrm{Cl}$ interval measurements were made for each subject. Sensitivities and specificities of proposed occipital condyle-Cl interval cutoffs of 2 and 3 SDs above the mean and previously published occipital condyle-Cl interval cutoffs for atlanto-occipital injury were then calculated on the basis of occipital condyle- $\mathrm{Cl}$ interval measurements for each subject.

RESULTS: An occipital condyle-C1 interval 2 SDs above the age-specific mean has a sensitivity of $50 \%$ and specificity of $89 \%-100 \%$, depending on the age group. An occipital condyle-Cl interval 3 SDs above the age-specific mean has a sensitivity of $50 \%$ and a specificity of $95 \%-100 \%$. A 4.0-mm occipital condyle-C1 interval has a sensitivity of $36 \%$ and a specificity of $100 \%$ in all age groups. A $2.5-$ mm occipital condyle- $\mathrm{Cl}$ interval has a sensitivity of $93 \%$ and a specificity of $18 \%-100 \%$.

CONCLUSIONS: Occipital condyle-Cl interval widening cutoffs used to establish atlanto-occipital injury lack both sensitivity and specificity in children and young teenagers. MR imaging is necessary to establish a diagnosis of atlanto-occipital injury in children and young teenagers when the appropriate mechanism of injury is present.

ABBREVIATIONS: $\mathrm{AOD}=$ atlanto-occipital dislocation; $\mathrm{CCl}=$ occipital condyle-Cl interval

$\mathbf{T}$ his is the second part of a 2-part study assessing the occipital condyle-C1 interval (CCI) in the pediatric population. Part 1 of the study established the normative values of the CCI for 7 pediatric age groups based on 124 patients who were imaged for indications other than trauma or developmental anomaly. ${ }^{1}$ Part 2 of this study aimed to determine the utility of applying age-specific abnormal CCI cutoff values to detect atlanto-occipital injury.

Prior studies have found that the CCI is a sensitive and specific marker for atlanto-occipital dislocation (AOD). ${ }^{2,3}$ Pang et $\mathrm{al}^{2}$ found a CCI cutoff of $4 \mathrm{~mm}$ to be $100 \%$ sensitive and specific for

Received September 4, 2015; accepted after revision October 31.

From the Departments of Radiology (B.C., L.L.L., J.L.L.) and Neurosurgery (S.V.), Cincinnati Children's Hospital Medical Center, University of Cincinnati School of Medicine, Cincinnati, Ohio.

Please address correspondence to Luke Linscott, MD, Cincinnati Children's Hospital Medical Center, 3333 Burnet Ave, ML 5031, Cincinnati, OH 45229; e-mail: luke.linscott@cchmc.org; @LukeLinscott

三 Indicates article with supplemental on-line table.

http://dx.doi.org/10.3174/ajnr.A4641
AOD in the pediatric population. Gire et $\mathrm{al}^{3}$ found a CCI cutoff of $2.5 \mathrm{~mm}$ for both the adult and pediatric populations to have a sensitivity of $100 \%$ and a specificity of $84 \%$ for AOD. These values assume that the CCI joint-space measurement is unchanged throughout childhood and early adolescence; this assumption is contrary to the findings in Part 1 of our study. Because we have evaluated pediatric subjects with trauma suspected of having AOD with concordant MR imaging evidence of atlanto-occipital injury, it has been our experience that the CCI joint space is not consistently widened in cases of atlanto-occipital injury. The purpose of this study was to determine the sensitivity and specificity for detection of atlanto-occipital injury by using age-specific abnormal CCI cutoff values derived from Part 1 of this study as well as the prior proposed abnormal cutoff values of 4 and $2.5 \mathrm{~mm}$.

\section{MATERIALS AND METHODS \\ Subject Selection}

This is a retrospective study approved by our institutional review board and performed at a large tertiary care children's hospital. 
Patients who had received the diagnosis of atlanto-occipital injury secondary to craniocervical trauma from 2004 to 2015 were identified by using a combination of radiology and clinical data bases. The radiology data base (Illuminate Softtech; https:/www. softekinc.com/illuminate/) was searched for the following terms: "atlanto-occipital dislocation," "atlanto-occipital dissociation," "craniocervical dislocation," "tectorial membrane," and "craniocervical fusion." All subjects in the clinical craniocervical trauma data base were also identified. Inclusion criteria were the following: 1) CT examination of the craniocervical junction at the time of injury with coronal and sagittal reconstructions, and 2) clinical or imaging evidence of atlanto-occipital injury. Clinical evidence of AOD is defined as an appropriate mechanism of injury for AOD with neurologic symptoms referable to the brain stem and the $\mathrm{C} 1-\mathrm{C} 2$ level. Imaging evidence of atlanto-occipital injury is defined as tectorial membrane rupture or displacement from the dorsal clivus on MR imaging or follow-up CT demonstrating ligamentous injury (ie, calcification within the tectorial membrane not present on the initial CT examination). The mechanism of injury and subject treatment (conservative therapy versus surgical craniocervical fusion) were tabulated.

\section{Image Analysis}

After subjects were identified, each imaging examination was reviewed by 2 investigators. The first examiner (L.L.L.) was an attending pediatric neuroradiologist with 8 years of experience interpreting MR imaging and CT in the context of craniocervical trauma. The second examiner (B.C.) was a fourth-year medical student who received instruction by the first examiner on how to appropriately make CCI measurements on a subset of cases. The same technique as in part 1 of the study was used to determine the average CCI for each joint space. ${ }^{1}$ This technique is similar to that used by Pang et al, ${ }^{2}$ except that sagittal localizer images were used to confidently exclude the medial occipital condyle notch, when present. ${ }^{1}$ The average CCI measurements made by the 2 examiners were averaged. In Part 1 of our study, the average sagittal CCI was found to have smaller SDs and interobserver variability. Thus, we chose the average sagittal CCI in the midjoint as our standard.

For a subject examination to be considered positive, the following definitions applied: For 2 and 3 SDs above the age-specific means from Part 1 of the study, any 1 of the 2 (right or left) average sagittal CCI measurements must be $\geq 2$ or 3 SD cutoffs. The age-specific sagittal plane cutoff values 2 and 3 SDs above the mean derived from Part 1 of this study are as follows: younger than 12 months (2.30, $2.60 \mathrm{~mm}), 12-24$ months (2.81, $3.12 \mathrm{~mm})$, $2-4$ years $(3.16,3.54 \mathrm{~mm}), 5-8$ years $(2.71,3.03 \mathrm{~mm}), 9-12$ years $(2.50,2.75 \mathrm{~mm}), 13-17$ years $(1.59,1.84 \mathrm{~mm})$, and older than 17 years $(1.20,1.35 \mathrm{~mm})$. For the standard of Pang et al, ${ }^{2}$ the average CCI (defined as the average of unilateral coronal and sagittal average CCI measurements) must be $\geq 4 \mathrm{~mm}^{2}$. For the standard of Gire et $\mathrm{al},{ }^{3}$ the "revised CCI" (defined as the largest single measurement on sagittal images in the midjoint) must be $\geq 2.5 \mathrm{~mm}^{3}$.

Initial CT images were also evaluated for qualitative findings suggesting atlanto-occipital injury, including retroclival epidural hematoma, subarachnoid hemorrhage in the perimedullary cistern, or perivertebral hemorrhage. MR images were reviewed for findings of major ligamentous injury at the craniocervical junc- tion, including tectorial membrane disruption or the tectorial membrane being separated from the clivus or odontoid.

\section{Statistical Analysis}

Sensitivities with 95\% CIs were calculated for each of the 4 abnormal cutoff standards by using the subjects with atlanto-occipital injury. Due to the small sample size, age-specific sensitivities could not be calculated. Age-specific specificities with 95\% CIs were calculated by applying the 4 abnormal cutoff standards to each individual subject from the subjects without trauma in Part 1 of the study (124 subjects in 7 different age groups). Sensitivities and specificities, including $95 \%$ CIs, were calculated by using the Wilson procedure without a correction for continuity at VassarStats (www.vassarstats.net).

\section{RESULTS}

Fourteen subjects ( 10 boys and 4 girls) $1-16$ years of age (mean, 6.9 years) met the inclusion criteria for the study. The On-line Table summarizes the following: subject demographics, CCI measurements for the right and left $\mathrm{OC}-\mathrm{C} 1$ joint spaces, interpretation of the study (positive versus negative) based on the 4 tested abnormal cutoffs, initial CT findings, follow-up MR imaging/CT results, mechanism of injury, clinical symptoms at presentation, and treatment.

With an abnormal average unilateral CCI cutoff of $4 \mathrm{~mm}$ proposed by Pang et al, ${ }^{2}$ 5/14 subjects would have a positive result, yielding a sensitivity of $36 \%$ (95\% CI, $0.16-0.61)$. With the revised CCI proposed by Gire et $\mathrm{al}^{3}{ }^{3} 13 / 14$ would have a positive result, generating a sensitivity of $93 \%$ ( $95 \%$ CI, 0.69-0.99). With a CCI cutoff of 2 and 3 SDs above the age-specific mean sagittal CCI from Part 1 of our study, 7/14 and 7/14 examinations would have positive results, yielding a sensitivity of 50\% (95\% CI, $0.27-$ 0.73 ) and 50\% (95\% CI, 0.27-0.73), respectively.

Specificities for each of the 4 cutoff values by age group calculated using healthy subjects from Part 1 of our study as controls are found in the Table.

Figures 1-3 show CT and MR images from 3 select cases (cases 10,3 , and 2) among the case series. Figure 4 shows the average sagittal and coronal CCI measurements in these same 3 cases compared with age-specific normative data.

\section{DISCUSSION}

In Part 1 of our series, concerning the normative CCIs in children and young adults, we found age-related variability in CCI measurements related to developmental dynamics of CCI morphology and increased prevalence of the medial occipital notch. Two earlier studies examined the normal CCI measurements in patients without AOD trauma and incorporated single-plane measurements, with one of the studies (Vachhrajani et $\mathrm{al}^{4}$ ) demonstrating a nearly 2-fold difference from that of Pang et al. ${ }^{5}$ Vachhrajani et al were the first to report age-related changes in the CCI, and we further confirmed that finding with our normative study by using the largest $100 \%$ nontraumatic subject group to date. ${ }^{1}$ In Part 2 of our study reported here, we examined the clinical presentation of children diagnosed with AOD after substantial trauma and symptoms referable to the brain stem and/or C1-C2 level deficits. As we began this study, it was our hope that 
Proportions of false-positive examination findings and resulting specificities with $95 \%$ Cls when applying the 4 proposed abnormal cutoffs to the 124 healthy subjects without trauma in Part 1 of the study

\begin{tabular}{|c|c|c|c|c|c|c|c|c|}
\hline \multirow[b]{2}{*}{ Age Group } & \multicolumn{4}{|c|}{$\begin{array}{l}\text { Proportion of False-Positive Examination } \\
\text { Findings }\end{array}$} & \multicolumn{4}{|c|}{ Specificities (95\% Cls) } \\
\hline & $\begin{array}{c}\text { Pang et } \mathrm{al}^{2} \\
(4 \mathrm{~mm})\end{array}$ & $\begin{array}{c}\text { Gire et } \mathrm{al}^{3} \\
(2.5 \mathrm{~mm})\end{array}$ & 2 SDs & 3 SDs & $\begin{array}{c}\text { Pang et } \mathrm{al}^{2} \\
(4 \mathrm{~mm})\end{array}$ & $\begin{array}{l}\text { Gire et } \mathrm{al}^{3} \\
(2.5 \mathrm{~mm})\end{array}$ & 2 SDs & 3 SDs \\
\hline Younger than $12 \mathrm{mo}$ & $0 / 21$ & $1 / 21$ & $1 / 21$ & $1 / 21$ & $1.0(0.85-1.0)$ & $0.95(0.77-0.99)$ & $0.95(0.77-0.99)$ & $0.95(0.77-0.99)$ \\
\hline $12-24 \mathrm{mo}$ & $0 / 19$ & $12 / 19$ & $1 / 19$ & $0 / 19$ & $1.0(0.83-1.0)$ & $0.37(0.19-0.59)$ & $0.94(0.73-0.99)$ & $1.0(0.83-1.0)$ \\
\hline $2-4 y r$ & $0 / 17$ & $14 / 17$ & $1 / 17$ & $0 / 17$ & $1.0(0.82-1.0)$ & $0.18(0.06-0.41)$ & $0.94(0.73-0.99)$ & $1.0(0.82-1.0)$ \\
\hline $5-8 \mathrm{yr}$ & $0 / 19$ & $13 / 19$ & $0 / 19$ & $0 / 19$ & $1.0(0.83-1.0)$ & $0.32(0.15-0.54)$ & $1.0(0.83-1.0)$ & $1.0(0.83-1.0)$ \\
\hline $9-12 \mathrm{yr}$ & $0 / 16$ & $3 / 16$ & $0 / 16$ & $0 / 16$ & $1.0(0.81-1.0)$ & $0.81(0.57-0.93)$ & $1.0(0.72-0.99)$ & $1.0(0.81-1.0)$ \\
\hline $13-17 \mathrm{yr}$ & $0 / 19$ & $0 / 19$ & $2 / 19$ & $0 / 19$ & $1.0(0.83-1.0)$ & $1.0(0.83-1.0)$ & $0.89(0.69-0.97)$ & $1.0(0.83-1.0)$ \\
\hline Older than $17 \mathrm{yr}$ & $0 / 13$ & $0 / 13$ & $1 / 13$ & $0 / 13$ & $1.0(0.67-1.0)$ & $1.0(0.67-1.0)$ & $0.92(0.67-0.99)$ & $1.0(0.67-1.0)$ \\
\hline
\end{tabular}

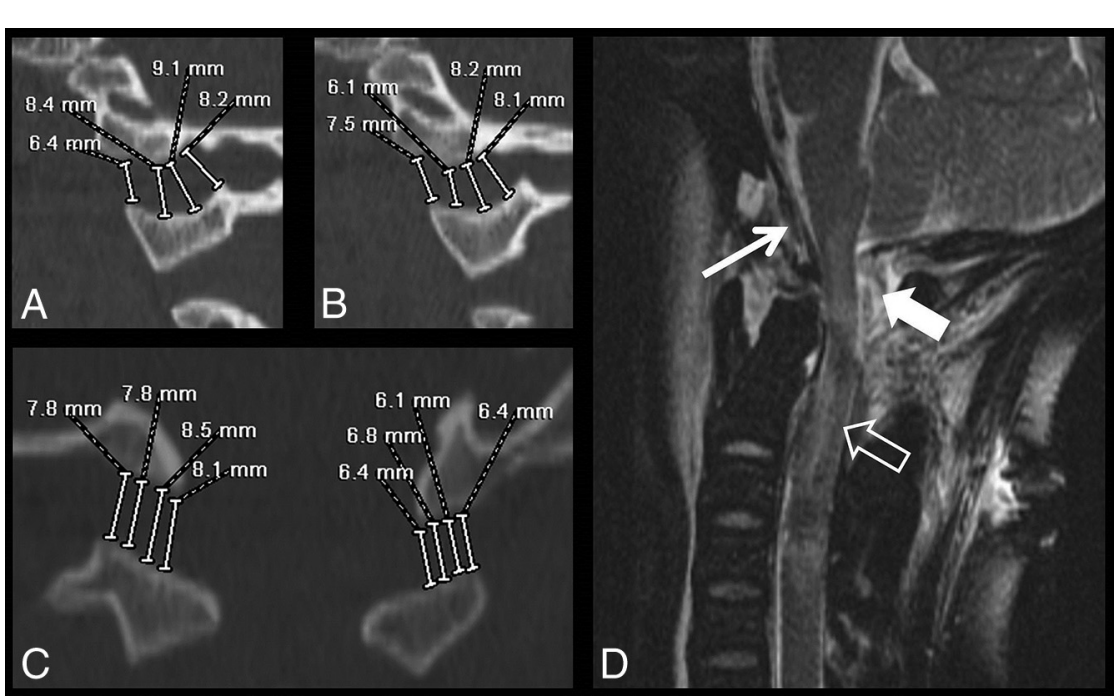

FIG 1. Subject 10. A 9-year-old girl involved in a motor vehicle collision. Initial sagittal (right, $A$; left, $B$ ) and coronal (C) CT images demonstrate substantial widening of the bilateral $\mathrm{CCI}$. MR imaging examination $(D)$ of the cervical spine performed 27 hours later demonstrates complete transection of the tectorial membrane (white arrow), complete transection of the posterior dural reflection at the craniocervical junction (block white arrow), and cord signal abnormality (open white arrow).

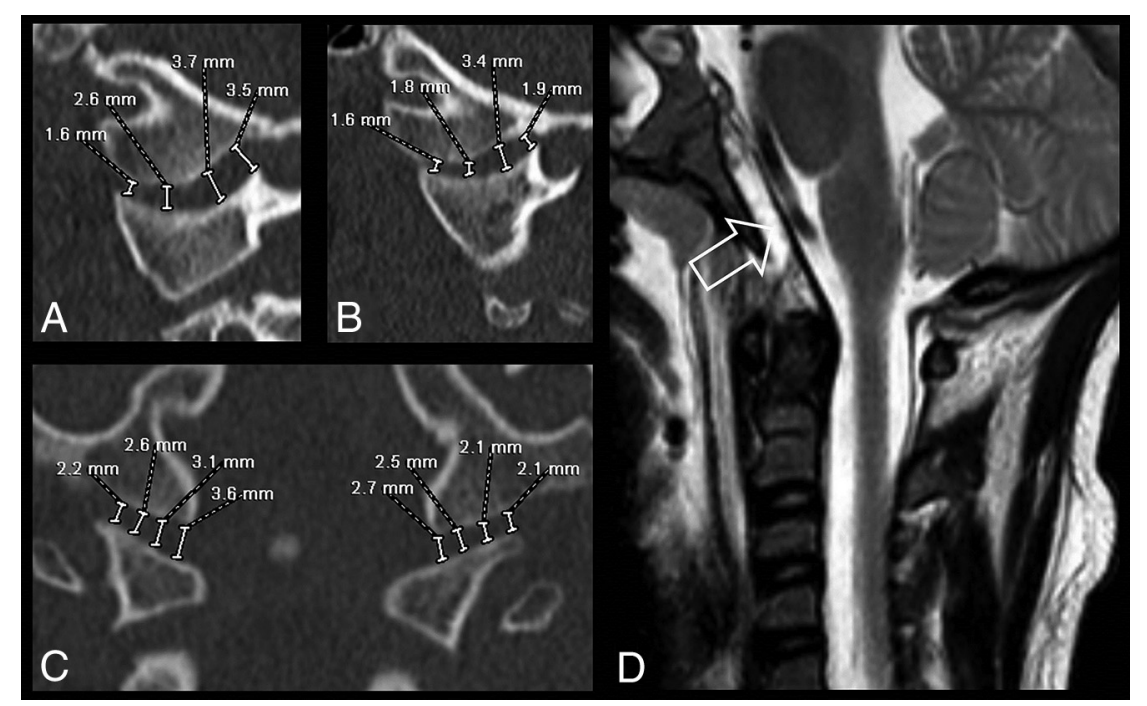

FIG 2. Subject 3. A 7-year-old girl involved in a motor vehicle collision. Initial sagittal (right, $A$; left, $B) \mathrm{CT}$ images demonstrate widening of the posterior $\mathrm{CCl}$ joint space, and coronal (C) images demonstrate mild asymmetry of the $\mathrm{CCl}$ joint spaces. Sagittal T2-weighted image of the cervical spine (D) performed 3 days later demonstrates lifting of the tectorial membrane off the dorsal clival cortex (open white arrow). applying abnormal CCI cutoff values based on age-specific normative data would provide us with an optimally sensitive and specific quantitative measure to detect atlanto-occipital injury in children.

Our data suggest that the mechanisms involved in atlanto-occipital injury do not consistently result in an enlarged CCI measure, particularly in cases of mild-to-moderate severity. The agespecific CCI cutoff values of 2 and 3 SDs above the mean, respectively, were selected to ensure a high specificity. While offering quite good specificity, the agespecific standard of 2 and 3 SDs above the mean CCI cutoff resulted in an unacceptably low sensitivity (50\% for both cutoff values) for atlanto-occipital injury in this case series. Our findings do not support the high sensitivities and specificities reported previously by Pang et al $(4 \mathrm{~mm})^{2}$ and Gire et al $(2.5 \mathrm{~mm}){ }^{3}$ The 4-mm average CCI abnormal cutoff value proposed by Pang et al does allow excellent specificity and avoids falsepositive results but has very poor sensitivity for atlanto-occipital injury. In contrast, the revised CCI method proposed by Gire et al with a single 2.5-mm measurement cutoff value has excellent sensitivity for atlanto-occipital injury but has a very low specificity in younger age groups. The revised CCI method would presumably result in a high falsepositive rate and low positive predictive values in the pediatric traumatic population, given the overall low incidence of atlanto-occipital injuries among all pediatric cervical spine trauma patients. ${ }^{6}$

We were surprised by the discrepant results we found in comparison with those of Pang et $\mathrm{al}^{2}$ regarding the sensitivity of their proposed 4-mm cutoff value. While these results may be at least 
partly due to variability in the imaging assessment, a careful comparison of subjects in each of these studies suggests that the clinical severity of injury is the most likely explanation. Overall, subjects in the article of Pang et al were more likely to have severe

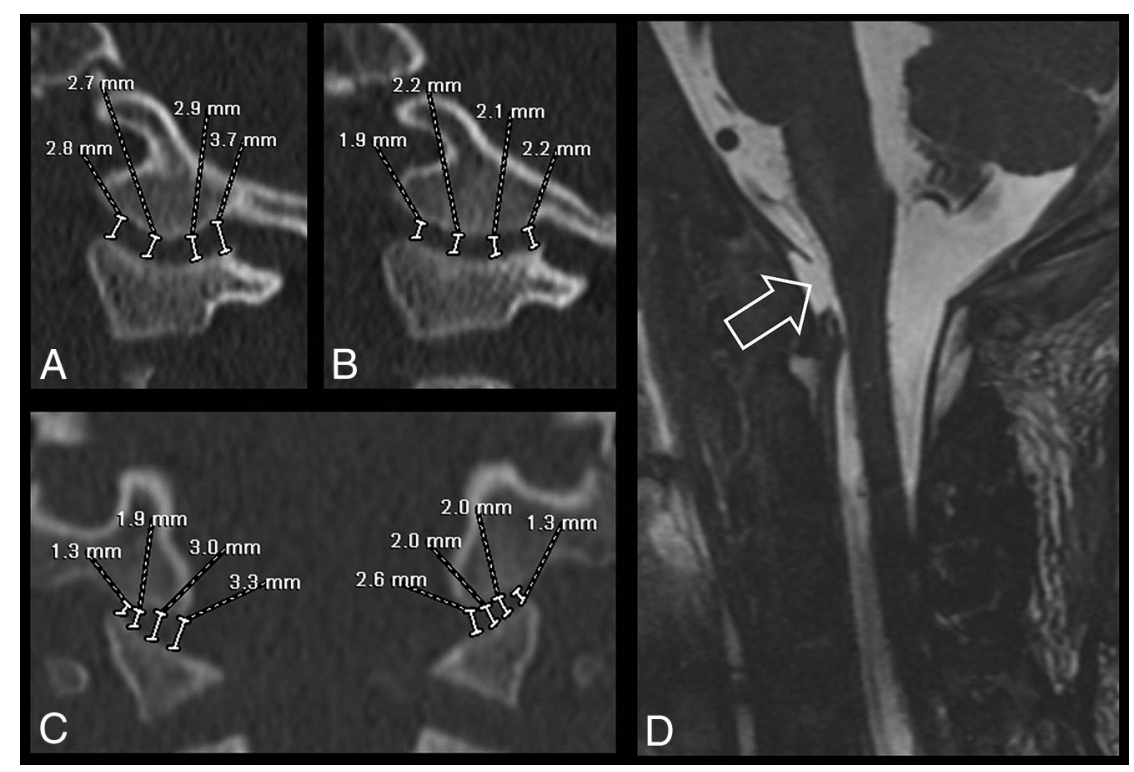

FIG 3. Subject 2. A 3-year-old boy hit by a car. Initial sagittal (right, $A$; left, $B$ ) and coronal (C) CT images demonstrate normal measurements of the bilateral CCI. MR imaging examination $(D)$ of the cervical spine performed 34 hours later demonstrates complete transection of the tectorial membrane (open white arrow).
C1-C2 neurologic deficits, while many of our subjects did not. This finding suggests that a 4-mm cutoff is likely highly sensitive and specific for severe cases of AOD but not for cases presenting with mild-to-moderate severity, which also may require surgical treatment.

The revised CCI 2.5-mm abnormal cutoff proposed by Gire et $\mathrm{al}^{3}$ appears to be highly sensitive as reported in their study. However, this measure would result in many false-positive findings in young children and young teenagers. For example, according to our normative data, most (82\%; 59\%-94\%) healthy subjects between 2 and 4 years of age would have single midjoint sagittal plane measurements of $>2.5 \mathrm{~mm}$, and these measurements would thus be considered abnormal and suspicious for atlanto-occipital injury. The Table illustrates the low specificity of the $2.5-\mathrm{mm}$ cutoff in subjects ages 1-12 years. A careful analysis of the subjects in the article of Gire et al provides a likely explanation for their reported high specificity (86\%). While many of the subjects with AOD in their study were young children, none of the control subjects used to cal-

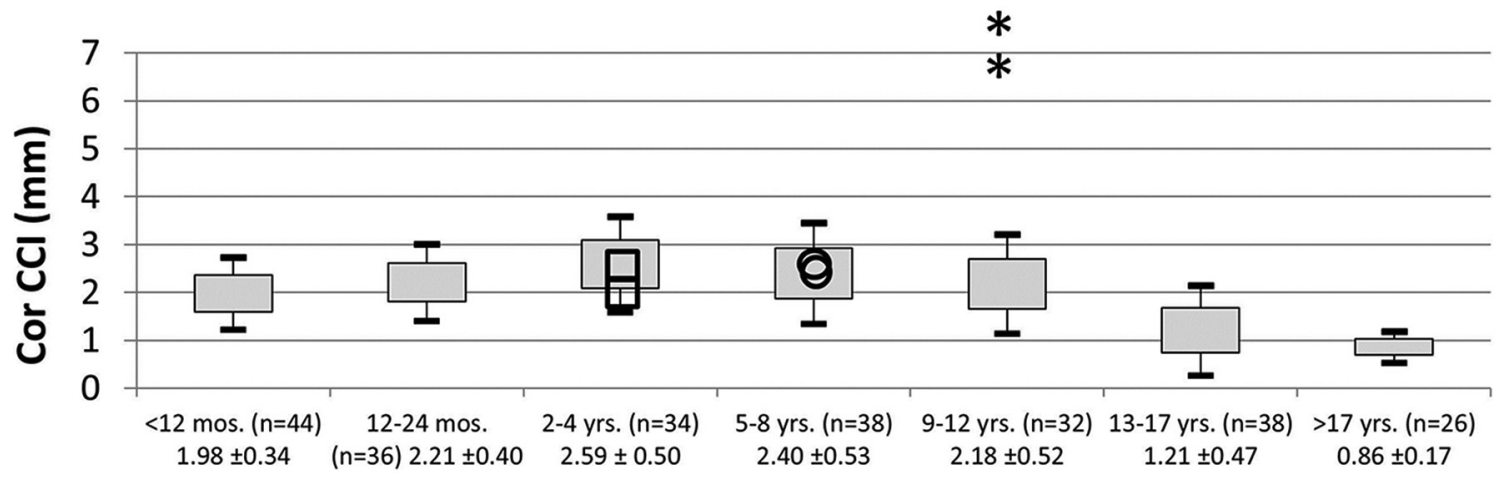

Age

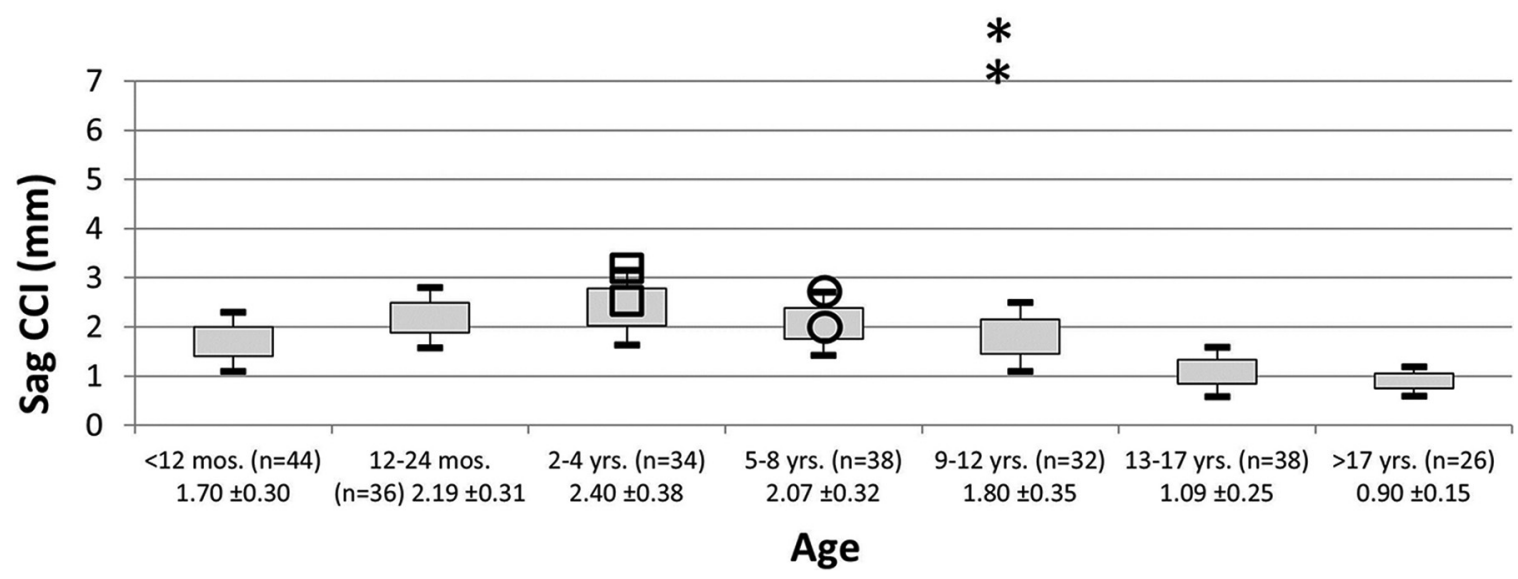

FIG 4. Representation of $\mathrm{CCl}$ measurements for subjects 10 (asterisks), 3 (circles), and 2 (squares) in relation to age-specific normative data from Part 1 of this study. Sag indicates sagittal; Cor, coronal. 
culate their specificity values were younger than 18 years of age. Thus, as confirmed in our study, the revised CCI cutoff value of $2.5 \mathrm{~mm}$ is likely highly sensitive and specific in subjects older than 18 years of age. On the basis of the results of Gire et al and this study, we do not recommend applying this cutoff value in subjects younger than 13 years of age.

While overall our results suggest that abnormal CCI measurements are somewhat limited in establishing a diagnosis of atlantooccipital injury, especially in mild-to-moderate cases, the CCI remains the best quantitative measure among many others (eg, Powers ratio, basion-dens interval, basion-axis interval, and so forth) for this diagnosis. ${ }^{2,7}$ Also, when we took all CT imaging findings into account, CT remains an excellent initial test for detecting injury at the CCI, with only $3 / 14$ (21\%) CT examinations in our study showing no evidence of injury at the craniocervical junction. CCI measurements should not be used in isolation, but rather in the context of other imaging and clinical findings.

The utility of MR imaging in the evaluation of acute craniocervical and cervical spine injury has been extensively studied in adults and shows little-to-no benefit after normal findings on CT evaluations of the cervical spine in neurologically intact patients. ${ }^{8-10}$ However, studies in children, who are much more susceptible to atlanto-occipital injury than adults, ${ }^{11,12}$ suggest a more essential role of MR imaging in the correct diagnosis of otherwise occult injuries at the craniocervical junction. ${ }^{13,14}$ In 1 study of subjects younger than 18 years of age, Junewick et $\mathrm{al}^{13}$ showed that 30/45 subjects with normal CT findings at the craniocervical junction had abnormal findings on MR imaging. Seventeen of these patients, most younger than 8 years of age, had findings that met the criteria for "significant craniocervical junction injury," though the need for surgical management was not investigated in this study. Another study by Meoded et $\mathrm{al}^{14}$ evaluated 10 subjects with traumatic retroclival epidural hematomas on CT who underwent MR imaging evaluation. They found that MR imaging was more sensitive for the detection of tectorial membrane disruption and recommended its use for complete evaluation of suspected atlanto-occipital injury in children. Our findings support the previous work of Junewick et al and Meoded et al in clearly identifying major differences in the value of MR imaging after initial CT imaging for children compared with adults with suspected atlanto-occipital injury.

Limitations of this study include its retrospective nature and relatively small sample size of atlanto-occipital injury. Small sample size is a limitation of many prior studies of atlanto-occipital dislocation. Larger multi-institutional studies examining the optimal diagnostic approach and management of this uncommon diagnosis would be helpful in arriving at a consensus. Some may question the authors' decision to include patients who did not undergo spinal fusion as part of the management of atlantooccipital injury. The threshold for surgical treatment of atlantooccipital injury varies across institutions and individual neurosurgeons. On the basis of the mechanism of injury, imaging findings, and clinical symptoms, we thought that these non- operative subjects were appropriately classified as having atlanto-occipital injury.

\section{CONCLUSIONS}

A substantial percentage of children with atlanto-occipital injury will have CCI measurements within the normal range for age. Age-specific abnormal CCI cutoff values and previously reported abnormal CCI cutoff values either lack sensitivity $(4 \mathrm{~mm})$ or specificity $(2.5 \mathrm{~mm})$ for less severe, but clinically significant, cases of atlanto-occipital injury. In children with clinical suspicion for atlanto-occipital injury but normal CCI measurements, MR imaging plays an important role in complete evaluation of these injuries.

\section{REFERENCES}

1. Smith P, Linscott LL, Vadivelu S, et al. Normal development and measurements of the occipital condyle- $\mathrm{C} 1$ interval in children and young adults. AJNR Am J Neuroradiol 2015 Oct 29. [Epub ahead of print] CrossRef Medline

2. Pang D, Nemzek WR, Zovickian J. Atlanto-occipital dislocation, part 2: the clinical use of (occipital) condyle-C1 interval, comparison with other diagnostic methods, and the manifestation, management, and outcome of atlanto-occipital dislocation in children. Neurosurgery 2007;61:995-1015; discussion 1015 CrossRef Medline

3. Gire JD, Roberto RF, Bobinski M, et al. The utility and accuracy of computed tomography in the diagnosis of occipitocervical dissociation. Spine J 2013;13:510-19 CrossRef Medline

4. Vachhrajani S, Sen AN, Satyan K, et al. Estimation of normal computed tomography measurements for the upper cervical spine in the pediatric age group. J Neurosurg Pediatr 2014;14:425-33 CrossRef Medline

5. Pang D, Nemzek WR, Zovickian J. Atlanto-occipital dislocation, part 1: normal occipital condyle-C1 interval in 89 children. Neurosurgery 2007;61:514-21; discussion 521 CrossRef Medline

6. Patel JC, Tepas JJ 3rd, Mollitt DL, et al. Pediatric cervical spine injuries: defining the disease. J Pediatr Surg 2001;36:373-76 CrossRef Medline

7. Horn EM, Feiz-Erfan I, Lekovic GP, et al. Survivors of occipitoatlantal dislocation injuries: imaging and clinical correlates. J Neurosurg Spine 2007;6:113-20 CrossRef Medline

8. Steigelman M, Lopez P, Dent D, et al. Screening cervical spine MRI after normal cervical spine CT scans in patients in whom cervical spine injury cannot be excluded by physical examination. Am J Surg 2008;196:857-62; discussion 862-63 CrossRef Medline

9. Schuster R, Waxman K, Sanchez B, et al. Magnetic resonance imaging is not needed to clear cervical spines in blunt trauma patients with normal computed tomographic results and no motor deficits. Arch Surg 2005;140:762-66 CrossRef Medline

10. Tomycz ND, Chew BG, Chang YF, et al. MRI is unnecessary to clear the cervical spine in obtunded/comatose trauma patients: the fouryear experience of a level I trauma center. J Trauma 2008;64: 1258-63 CrossRef Medline

11. Junewick JJ. Pediatric craniocervical junction injuries. AJR Am J Roentgenol 2011;196:1003-10 CrossRef Medline

12. Junewick JJ. Cervical spine injuries in pediatrics: are children small adults or not? Pediatr Radiol 2010;40:493-98 CrossRef Medline

13. Junewick JJ, Meesa IR, Luttenton CR, et al. Occult injury of the pediatric craniocervical junction. Emerg Radiol 2009;16:483-88 CrossRef Medline

14. Meoded A, Singhi S, Poretti A, et al. Tectorial membrane injury: frequently overlooked in pediatric traumatic head injury. AJNR Am J Neuroradiol 2011;32:1806-11 CrossRef Medline 